\title{
An Efficient Markov Chain Based Channel Model for 6G Enabled Massive Internet of Things
}

\author{
Wei Yang1,2*, Xiaojun Jing ${ }^{3}$, Hai Huang ${ }^{3}$, Chunsheng Zhu ${ }^{4,5}$, Qiaojie Jiang ${ }^{2}$, Dongliang Xie ${ }^{1}$ \\ ${ }^{1}$ State Key Laboratory of Networking and Switching Technology, Beijing University of Posts and \\ Telecommunications, Beijing 100876, China \\ [E-mail: yangweibupt@bupt.cn] \\ ${ }^{2}$ Guangdong Southern Planning \& Designing Institute of Telecom Consultation Co., Ltd., Shenzhen 518038, China \\ ${ }^{3}$ School of Information and Communication Engineering, Beijing University of Posts and Telecommunications, \\ Beijing 100876, China \\ ${ }^{4}$ Institute of Future Networks, Southern University of Science and Technology, Shenzhen 518055, China \\ ${ }^{5}$ Research Center of Networks and Communications, Peng Cheng Laboratory, Shenzhen 518055, China \\ *Corresponding author: Wei Yang \\ Received July 6, 2021; revised September 27, 2021; accepted October 9, 2021; \\ published November 30, 2021
}

\begin{abstract}
Accelerated by the Internet of Things (IoT), the need for further technical innovations and developments within wireless communications beyond the fifth generation (B5G) networks is up-and-coming in the past few years. High altitude platform station (HAPS) communication is expected to achieve such high levels that, with high data transfer rates and low latency, millions of devices and applications can work seamlessly. The HAPS has emerged as an indispensable component of next-generations of wireless networks, which will therefore play an important role in promoting massive IoT interconnectivity with 6G. The performance of communication and key technology mainly depend on the characteristic of channel, thus we propose an efficient Markov chain based channel model, then analyze the HAPS communication system's uplink capability and swing effect through experiments. According to the simulation results, the efficacy of the proposed scheme is proven to meet the requirements of ubiquitous connectivity in future IoT enabled by 6G.
\end{abstract}

Keywords: Internet of Things, channel model, HAPS, cell capacity, quasi-stationary state

A preliminary version of this paper appeared in IEEE ICCT 2020, October 28-31, Nanning, China. This version includes a concrete analysis of the factors that affect uplink capacity as well as the effects of swinging. This research was supported by a research grant from the China Scholarship Council [20173109], the National Natural Science Foundation of China [61901049]. We express our thanks to Dr. Dinh Han Nguyen who checked our manuscript. 


\section{Introduction}

$\mathbf{I}_{\mathrm{n}}$ recent years, there is an explosion in the number of mobile users, with the development of economy and technology, the end-users expect more immediate and simple connection to internet and multimedia [1]. The smart city's construction, meanwhile, has come to a key stage. The compact and diverse system located in the urban area will accumulate exabytes (EB) of data to be shared between them [2]. By 2030, wireless data traffic is expected to exceed $4394 \mathrm{~EB}$, while $5 \mathrm{G}$ will not be able to support most of these advanced applications. Driven by massive and data intensive applications such as VAR gaming supported by IoT, B5G networks increasingly need technical innovation and advances in wireless connectivity [3]. Here, 6G is expected to extend its capabilities to keep pace with time. Therefore, extensive exploratory research has been conducted by a respectable quantity of related scholars and researchers. Among them, HAPS communication has been widely concerned by many scholars and scientists. Because, compared with satellite communication, it saves costs and has a broader achievements. In addition, as a hopeful technology, HAPS communication can realize the ubiquitous IoT connectivity, which may be the tendency of 6G technology development.

Following a literature review of HAPS communication and intensive IoT, it can be concluded that a large quantity of approaches, including fading and channel model, capacity estimation, as well as quasi-static state analytics, have been proposed and researched. In [4], the authors provide a vision and framework for the HAPS networks of the future supported, and present the radio resource management along with synergistic physical layer techniques. Besides, relative to Rice flat fading channel model, the ratio of multipath to direct component is higher [5]. In order to establish a HAPS ground user channel model in view of the multipath fading, it is also important to consider the selective frequency of the channel. In addition, an optimization method for the cell configuration for HAPS mobile communications using a genetic algorithm is proposed in [6]. The beam interference induced by platform rotation could be avoided by this algorithm, thereby effectively increasing the capacity of the device and enhancing the utilization of the spectrum. M. Guan et al. [7] estimate the downlink capacity of HAPS-CDMA system considering the relationship between beam coverage and spectral efficiency. HAPS could only be sustained in a quasi-static state because of the stratospheric wind [8], K. Hoshino et al. [9] believe that the HAPS in the vertical direction has greater side effect on the system capacity and larger cell soft handover than the displacement in the horizontal direction. The influence of HAPS displacement on the performance of the system under two separated antenna mechanisms is discussed in [10].

For the research of communication systems, channel modeling plays an important role. The current research on channel model of the stratospheric platform communication system mainly focus on the issues as follows, A. M. Al-Awadi and M. J. Al-Dujaili [11] used a semi-Markov process to study stratospheric communication channels. According to different coverage positions of the stratospheric platform, the channels were divided into villages, suburbs, and urban areas to meet different probability distributions, the result of switching between Lee, normal and Rice distribution was analyzed. S. Zhang et al. [12, 13] studied the communication link between the stratospheric platform and the ground mobile station, and considered the multipath fading. It is believed that when the multipath component accounts for a large proportion of the direct component, or information rate is high, the Rice channel could not be simply used to approximate the HAPS channel. In accordance with the 6G expectations, 
K.Tekbıyık et al. in [14] consider a HAPS mounted reconfigurable intelligent surface-assisted two-way communications and obtain a low overhead and a high normalized mean square error performance.

HAPS is affected by the stratospheric wind and could only be maintained in a quasi-static state, [15] gives a quasi-stationary model in HAPS working state, indicating that the platform moves within the range of vertical $\pm 700 \mathrm{~m}$ and horizontal $\pm 400 \mathrm{~m}$, and swings within the range of angle less than $1^{\circ}$. O. Anicho et al. [16] believes that the displacement in the vertical direction is more prominent than the displacement in the horizontal direction in terms of system capacity and cell soft handover. C. Queiroz et al. [17] discusses the impact of platform displacement on system performance under two multi-antenna mechanisms. Above literatures are based on the following two assumptions, that is, when the platform is unstable, the user can switch immediately without dropped calls. On the other hand, only the impact of platform displacement on system performance is considered, and it is believed that the platform swing has a small angle on the system while its impact on capacity could be negligible. Therefore, the simulation conclusions have certain limitations compared with the real scenario.

To meet the challenges mentioned above, we proposed an efficient Markov chain-based channel model in HAPS for massive IoT, and then the changes in uplink system capacity of the cells in different locations of stratospheric CDMA system are analyzed. Moreover, the influence of platform swing on system performance is analyzed as well as the platform swing model is proposed. It is known from the simulation results that the platform swing has a great impact on the system performance and cannot be ignored, while the proposed scheme has a good effect on improving this impact. To sum up, the main contributions of this paper could be summarized as the following three aspects.

- Firstly, considering that the coverage area of HAPS platform in actual communication environment is much larger than that of the terrestrial communication system, according to the receiver communication link's minimum elevation angle requirements, the HAPS coverage area is divided into three concentric zones. After that, a Markov chain-based channel model is established on HAPS communication which could support large-scale IoT communications in future 6G.

- Additionally, the HAPS system using CDMA technology is studied, and the system capacity under CDMA single access technology as well as the factors that affect HAPS-CDMA uplink capacity are analyzed in detail.

- Furthermore, the effects of swinging on HAPS system are analyzed when the instability platform in quasi-static state. Besides, to cope with the swing effect caused by wind influence in a small angle, we established a platform swing model which confirmed the influence of platform's small angle swing on the capacity of HAPS communication system.

For the rest, Section 2 reviews the characteristic of stratospheric channel along with HAPS communication network type, and subsequently proposes an efficient Markov chain-based channel model for massive IoT. Section 3 analyzes the capacity of HAPS communication system under CDMA single access mechanism, and then focus the platform swing influence in a small angle on the system link capacity. Section 4 includes numerical experiments and analysis of the efficiency of the proposed solution. Finally, Section 5 consists of a conclusion for this paper. 


\section{Channel Model in Stratospheric Space}

Recently, growing concern has been paid to HAPS which is a method of communication that emploies the aerial platform [18] installed in the lower stratosphere, at an altitude of about 20 kilometers and equipped with a valid communication payload. The structure diagram is shown in Fig. 1.

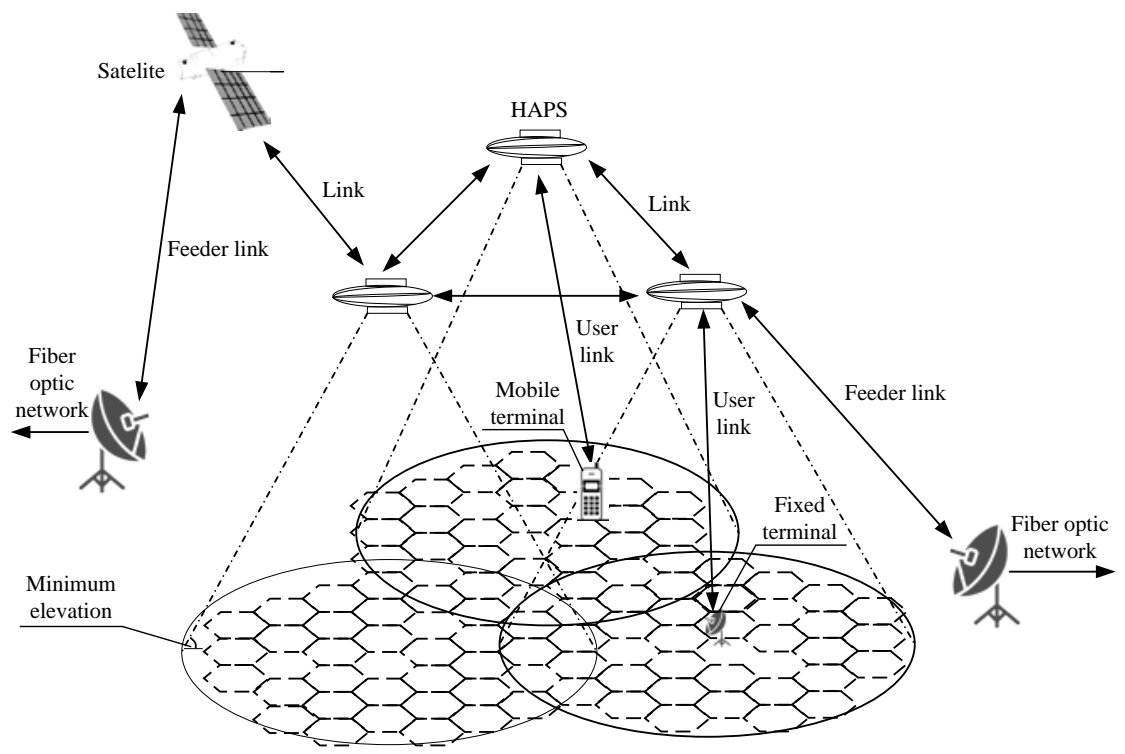

Fig. 1. Structure diagram of HAPS communication system

Fig. 1 indicates a framework for the stratospheric communication system, as is shown in the diagram, it comprises of three parts, that is, the ground section, the aerial section of the platform and the IoT user terminal equipment. For one thing, HAPS could be competent for communication with ground wireless terminals or ground control equipment [19]. For another thing, with the optical links, it can form a complete integrated mobile communication network system between platforms or between satellites and platforms. In addition, a single platform can use multi-beam antennas for ground wireless coverage. Each region covered by the beam is equal to a realistic cell, allowing wireless channels to be reused by spatially separated IoT users. In many ways, the HAPS communication system is equivalent to the ground cellular communication system and the satellite communication system, but it also has many special benefits. The communications system of the HAPS, with its specific atmospheric altitude between satellite and ground communication system has technological advantages that would lead to extensive use in the next few years.

\subsection{Network Type}

During the actual design and construction of HAPS communication system, it could be separately networked to provide wireless access services, and also could be networked with the ground mobile communication system to ensure reliable communication. Besides, it could be also used as a satellite communication relay node to form a complete air integration network. Specifically, these three networking modes correspond to different application scenarios, which will be described in detail as follows. 
The separate networking of HAPS could be applied to areas with relatively small numbers of users such as suburbs, at this time, it is difficult to implement the deployment of terrestrial cellular base stations. The utilization of satellite communication systems is expensive, however, the effect is not obvious. By using a helium-filled motorboat or a solar-powered aircraft as a carrier of a HAPS communication base station, each platform base station could cover an area. Within each area, wireless coverage is achieved by cellular projection of the ground through a multi-beam antenna installed on the platform, so that users with spatial separation are able to reuse wireless channels. At the same time, HAPS could also communicate through optical links. Multiple platforms form a communication system, providing a larger range of communication coverage networks and wireless access for ground IoT users. The HAPS base stations could communicate with ground control equipment [20], information interface equipment, and various types of wireless user terminals through the signaling station, the typical stratospheric separate network coverage system model is shown in Fig. 2.

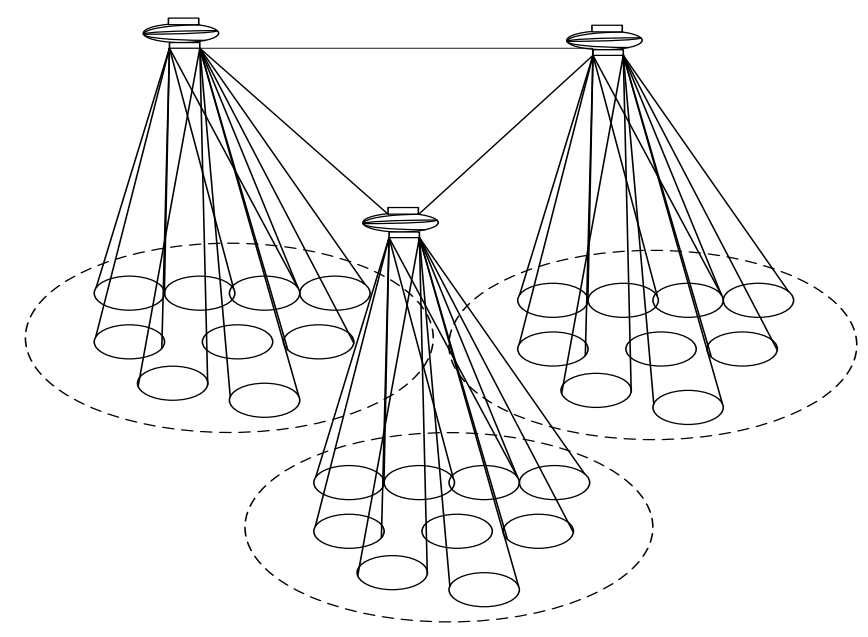

Fig. 2. Separate network for HAPS communication system

For hotspots with large user demand such as urban areas, individual networking can no longer satisfy the business needs of these areas. At this point in time, joint with HAPS could be used for cooperative networking. The HAPS platform projects a large cell, that is, a macro cell, to the ground through an antenna, and provides services for terminals with low data rate requirements. At the same time, terrestrial cellular base stations project micro-cells in various hotspot coverage areas to provide services for terminals with high data transmission rates. HAPS base stations are also connected and communicated through optical links, while HAPS and ground base stations are connected through ground gateways, the typical networking scheme of HAPS and ground cellular could be illustrated as Fig. 3. 


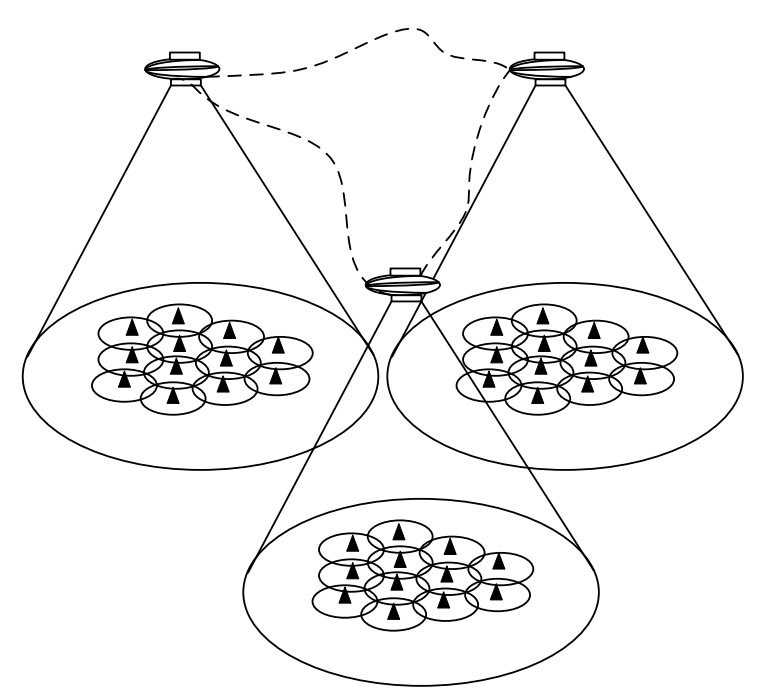

Fig. 3. Hybrid network of HAPS with ground cellular system

As a relay node for satellite communications, HAPS could be combined to form a complete air-ground-air integrated system. This kind of networking method makes full use of the advantages of three terrestrial cellular systems, while HAPS platform and satellite communication could provide users with high-quality communication and seamless access for terrestrial wireless terminals. Satellite communication systems could achieve large-scale coverage worldwide, and the HAPS platform, as a relay and forwarding station, could achieve high-speed access and multimedia communications within the system's coverage. HAPS communication system forms links through optical connections, and then uses specific algorithms to achieve union with satellites. The cellular coverage mode of HAPS and ground base stations is similar to macro-microcell embedded coverage mode, which can better meet the actual needs of data transmission rates and bandwidth between communication systems, and the typical HAPS-ground cellular-satellite coverage common networking scheme is shown in Fig. 4.

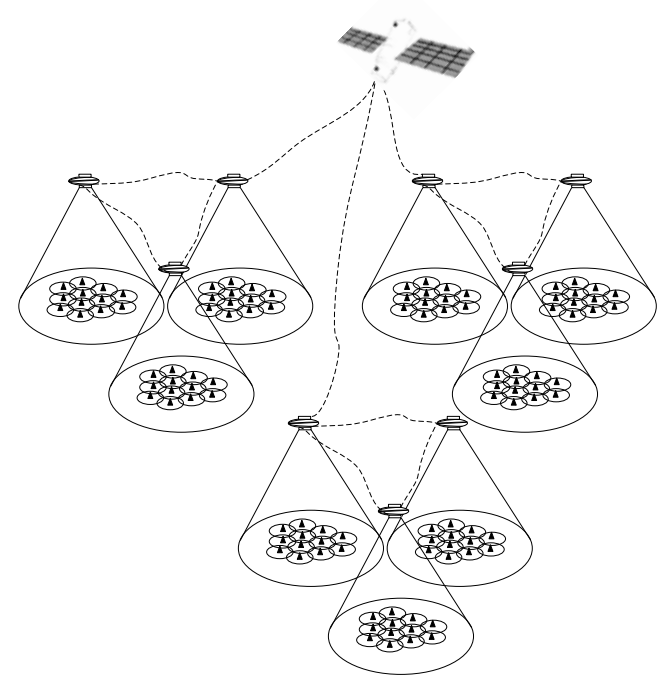

Fig. 4. Hybrid network of HAPS with ground cellular and satellite 


\subsection{Markov Chain-Based Channel Model}

The channel features of HAPS [21], ground mobile and satellite communication system are not only interrelated but also differ from each other. With specific geometric transmission structure and large coverage of the stratospheric platform, the HAPS couldn't be simulated by a single channel environment in actual stratospheric communication channel simulation. Depending on the elevation between the ground user and the HAPS station, the coverage area is separated into several circumstances, as shown in Fig. 5.

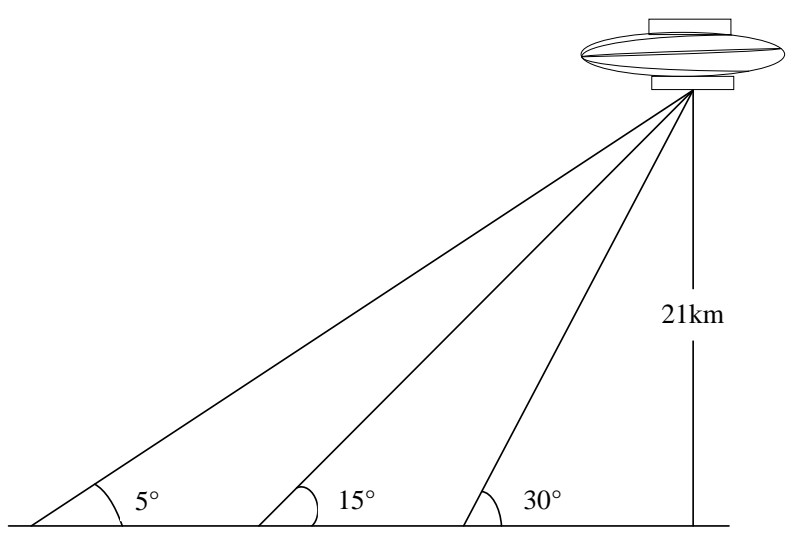

Fig. 5. Schematic diagram of HAPS coverage area division

As shown in Fig. 5, with different elevation angle of the receiver on the ground, the coverage area can be divided into urban area $\in\left(30^{\circ}, 90^{\circ}\right]$, suburbs $\in\left(15^{\circ}, 30^{\circ}\right]$ and forest $\in\left(5^{\circ}, 15^{\circ}\right]$. Theoretically, it would be possible to split the continuous environment of each area into $N$ relatively stable small region. When $N \rightarrow \infty$, the description of environment is the most accurate and detailed. When the communication angle is relatively large, the small-scale fading can be ignored. On the contrary, when the elevation angle is relatively small, apart from presence of strong DC components, the landform or surface construction may also lead to multipath effects. The envelope distribution of small-scale fading complies with the Rice distribution if there is a primary stable signal, such as line-of-sight transmission [22]. Therefore, the use of Rice channel model only simulates the HAPS wireless channel with small elevation angle.

The best way for channel characteristics research is to test and analyze the channel performance in actual communication environment, however, the real time testing and data collection are almost impossible for stratospheric communication platforms. Therefore, researchers usually use probability distribution models to simulate the channel environment of HAPS communication in reality. According to differences in shadowing degree of the actual signal, the channel status could be divided into three states, which satisfying three different probability density functions respectively. Subsequently, a three state switching channel model based on Markov chain is established, which is a simulation platform for the entire stratospheric communication system while its setup provides a more accurate simulation of transmission characteristics. With discrete states and time, the Markov process constitutes a Markov chain, the state of each moment is only connected to that of the previous moment, as shown in Fig. 6. 


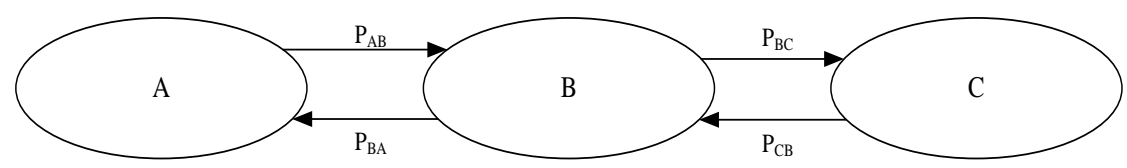

Fig. 6. Markov chain based channel model

The Markov model is a mathematical abstract model that believes that the state of a signal at a certain moment is only affected by the signal state of the adjacent moment, and has nothing to do with the signal state at other moments, which consistent with the random and slowly changing characteristics of channel environment. Besides, the Markov channel model is suitable for a wide range of non-static environments, where each state is described by a static model, and each static model satisfies different statistical probability characteristics. There are two key points in this statistical channel modeling, namely, the selection of static models and the switching criteria between different static models. In fact, the state exchange in various environmental areas [23] could be illustrated as Table $\mathbf{1}$ under different scenarios.

Table 1. A state transition in different environments

\begin{tabular}{|c|c|c|c|}
\hline $\begin{array}{c}\text { Transition } \\
\text { probability }\end{array}$ & $\begin{array}{c}\text { Urban } \\
\text { area }\end{array}$ & $\begin{array}{c}\text { Suburban } \\
\text { district }\end{array}$ & $\begin{array}{c}\text { Forest } \\
\text { area }\end{array}$ \\
\hline$P_{A B}$ & 1 & 1 & 1 \\
\hline$P_{A C}$ & 0 & 0 & 0 \\
\hline$P_{B A}$ & 0.65 & 0.65 & 0.42 \\
\hline$P_{B C}$ & 0.35 & 0.35 & 0.58 \\
\hline$P_{C A}$ & 0 & 0 & 0 \\
\hline$P_{C B}$ & 1 & 1 & 1 \\
\hline
\end{tabular}

As shown in Table 1, in the suburbs, the three states $A, B$ and $C$ of channel transition matrix $P$ could be expressed as

$$
P=\left[\begin{array}{lll}
P_{A A} & P_{A B} & P_{A C} \\
P_{B A} & P_{B B} & P_{B C} \\
P_{C A} & P_{C B} & P_{C C}
\end{array}\right]=\left[\begin{array}{ccc}
0 & 1 & 0 \\
0.65 & 0 & 0.35 \\
0 & 0 & 1
\end{array}\right]
$$

According to the convergence of the Markov process, given the state transition matrix $P$, the steady state probability matrices $\pi=\left(\pi_{A}, \pi_{B}, \pi_{C}\right)$ of the three states $A, B$ and $C$ could be derived as follows

and

$$
\pi P=\left(\pi_{A}, \pi_{B}, \pi_{C}\right)\left[\begin{array}{lll}
P_{A A} & P_{A B} & P_{A C} \\
P_{B A} & P_{B B} & P_{B C} \\
P_{C A} & P_{C B} & P_{C C}
\end{array}\right]=\pi=\left(\pi_{A}, \pi_{B}, \pi_{C}\right)
$$

$$
\pi e=1
$$

In accordance with the formula (2) (4), it is evident that the steady state matrix $\pi=(0.325,0.5,0.175)$ of the three states of $A, B$ and $C$ in suburbs could be obtained. 
Similarly, under the forest environment, the channel transition matrices $P$ could be illustrated as

$$
P=\left[\begin{array}{lll}
P_{A A} & P_{A B} & P_{A C} \\
P_{B A} & P_{B B} & P_{B C} \\
P_{C A} & P_{C B} & P_{C C}
\end{array}\right]=\left[\begin{array}{ccc}
0 & 1 & 0 \\
0.42 & 0 & 0.58 \\
0 & 0 & 1
\end{array}\right]
$$

In accordance with calculation, the steady state matrix of $A, B$ and $C$ states are $\pi=(0.21,0.5,0.29)$.

According to the situation that signals are blocked, the change in the quality of HAPS wireless channel is described as three different states $A, B$ and $C$. Among them, $A$ is in the LoS state, while direct and multipath components of the received signal are free from shadowing effect, the channel transmission characteristics are equivalent to random variables obeying Rice distribution. Besides, $B$ is partially shielded. The direct component of the received signal is affected by shadow occlusion, while the multipath component is immue from Rayleigh distribution. The transmission characteristics of the entire channel can be equivalent to the sum of two random variables, which satisfy the lognormal process and Rayleigh process. Furthermore, $C$ is completely shielded. The direct and multipath components of received signal are both affected by shadow occlusion, the channel transmission characteristics are equivalent to the product of two random variables that satisfy lognormal process and Rice process. The block diagram of the Markov channel structure of the three states switching in the HAPS is shown in Fig. 7.

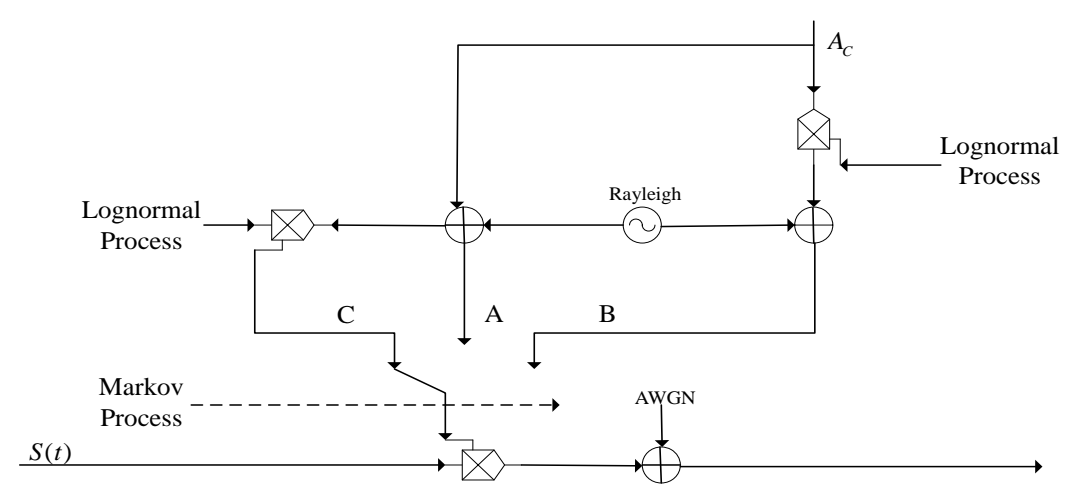

Fig. 7. Markov chain-based channel structure diagram

As shown in Fig. 7, $S(t)$ represents the input signal while the three states of $A, B$ and $C$ satisfy the following conditions. In the state $A$, the multipath signal component formed by the reflected wave caused by buildings, trees, or other reflective objects and the direct wave signal component $A_{C}$ are superposed. Generally speaking, the channel model is affected by factors such as relative position of the stratospheric platform and mobile terminal, as well as the angle of communication. Therefore, it is proposed to divide the coverage area of HAPS base station on the ground into three parts, that is, urban area, suburban area and forest area. Under each environment area, the proportions of $A, B$ and $C$ states duration are different. Specifically, due to the high-rise buildings in the urban environment, the probability of the signal being blocked is large. At this point, the direct component and the multipath component are affected by the shadow blocking. However, in the suburban and forest environments, the 
envelope follows the Rice distribution because the direct component and the multipath component are superimposed and not obscured by shadows.

\section{HAPS System Capacity}

In the hybrid networking of HAPS and ground system or satellite communication system, the shared bandwidth maybe result in interference [24], which will reduce the system capacity to some extent. In particular, when the HAPS communication platform forms a macro cell to cover the entire area, and the ground base station forms a micro cell to cover the hotspot area, the larger inter-system interference will bring about the reverse link capacity of entire system to decrease rapidly. This paper mainly focuses on the scenario that HAPS system is separately networked for CDMA single access. In this section, we first analysis the uplink capacity of HAPS system, and then discuss the capacity calculation in a static state. For efficient reference, important symbols are summarized in Table 2.

Table 2. Key parameters

\begin{tabular}{|c|c|}
\hline Notation & Meaning \\
\hline$i$ & User $i$ \\
\hline$K$ & Total number of cells \\
\hline$N$ & Number of cells \\
\hline$R_{b}$ & Information transmission rate \\
\hline$E_{b}$ & Bit energy of the signal \\
\hline$W$ & Total band width \\
\hline$N_{0}$ & Noise power spectral density \\
\hline$W / R_{b}$ & Processing gain of the system \\
\hline$E_{b} / N_{0}$ & Normalized signal-to-noise ratio \\
\hline$\eta$ & Background thermal noise \\
\hline$d$ & Duty cycle \\
\hline$m$ & Number of users accessing the system \\
\hline$r_{i j}$ & Path length \\
\hline$S_{i j}$ & Shadow fading \\
\hline$R$ & Cell radius \\
\hline$S$ & Cell area \\
\hline$G\left(\theta_{i j}\right)$ & Decimal expression of antenna gain \\
\hline$I_{0}$ & Total interference power \\
\hline$\sigma_{n}^{2}$ & Variance of Gaussian noise \\
\hline$f$ & Interference factor \\
\hline$T$ & The area covered by a cluster \\
\hline$I_{\text {int } r a}$ & Interference within cell \\
\hline$I_{\text {inter }}$ & Inter-cell interference \\
\hline$\Delta \theta(t)$ & Platform swing angle \\
\hline$P_{T}$ & Transmission power \\
\hline
\end{tabular}




\subsection{Honeycomb Structure with HAPS}

When stratospheric platforms are separately networked, multiple beams are used to project the cellular structure on the ground, and different cells could reuse frequency to increase system capacity. Assume that the HAPS platform uses equal-beam area multi-beam antennas to form regular hexagonal cells on the ground [25], as shown in Fig. 8.

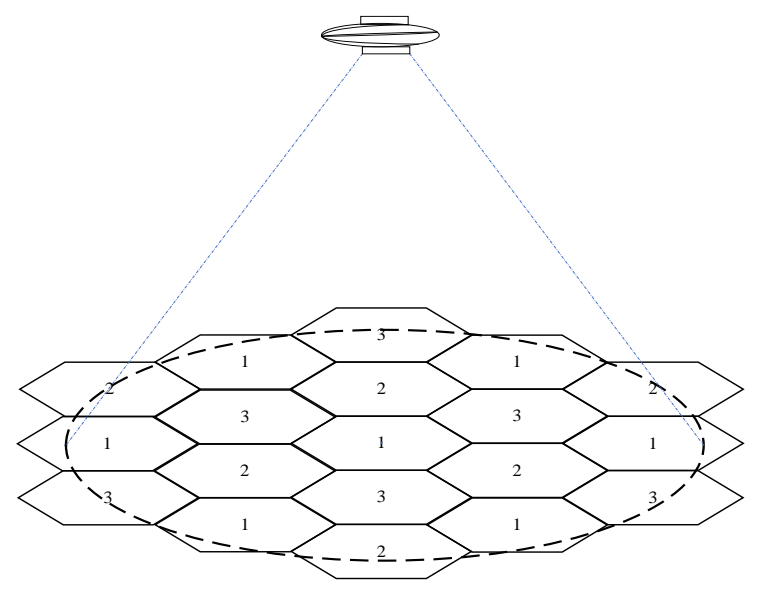

Fig. 8. HAPS cellular multiplexing structure

Fig. 8 shows a cellular structure model with a reuse factor of 3 in the coverage area, channels with the same number use the same frequency channel resources. In general, the total number of cells $K$ in the system is related to the number of cells $N$, and the relationship between them can be expressed as

$$
K=3 N(N-1)+1, N=1,2,3, \cdots
$$

In an actual HAPS communication system, the number of cells in the coverage area of the platform can reach dozens or even hundreds.

\subsection{HAPS System with CDMA}

In the HAPS system, it is assumed that the system is an interference-limited and noise-limited system. Before analyzing the uplink capacity of the system, we first introduce the HAPS system model, which is located at an altitude of $21 \mathrm{~km}$ and covers the service area for communication. Besides, the CDMA base station is located on the stratospheric platform. The structure of a common stratospheric communication system is shown in Fig. 9.

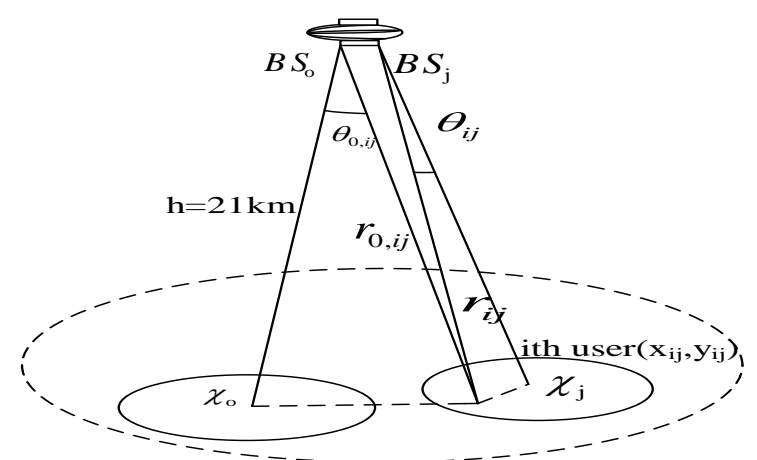

Fig. 9. HAPS communication system model 
The platform shown in Fig. 9 uses a multi-beam array antenna to cover the ground cell, and the center of the main beam is aligned with the center of the ground cell. Among them, the center coordinate of the reference cell is $(0,0)$, the center coordinate of the $j$ th cell is $\left(x_{j}, y_{j}\right)$, and the coordinate of the user $i$ in the $j$ th cell is $\left(x_{i j}, y_{i j}\right)$. Among them, $\theta_{0, i j}$ and $\theta_{i j}, r_{0, i j}$ and $r_{i j}, \varsigma_{0, i j}$ and $\varsigma_{i j}$ represent the angle, path length, and shadow fading of the main beam center of user $i$ with the center reference cell base station and serving cell base station, respectively. Since the path loss and shadow fading are basically the same, we can get that $r_{0, i j} \approx r_{i j}, \varsigma_{0, i j} \approx \varsigma_{i j}$. The gain of beam side lobes follows ITU standard [26]. Specifically, it could be expressed in $\mathrm{dB}$ as

$$
G(\theta)=\left\{\begin{array}{cc}
34.8-3\left(\frac{\theta}{1.57}\right)^{2} & 0^{\circ} \leq \theta \leq 4.53^{\circ} \\
9.8 & 4.53^{\circ}<\theta \leq 5.87^{\circ} \\
55.95-60 \log (\theta) & 5.87^{\circ}<\theta \leq 37^{\circ} \\
-38.2 & 37^{\circ}<\theta \leq 90^{\circ}
\end{array}\right.
$$

where $\theta$ stands for the angle off main beam center, and the central reference cell $B_{0}$ is located directly below HAPS, $21 \mathrm{~km}$ away from the platform. The other cells $B_{j}$ are distributed around the central reference cell in a honeycomb layered structure with a cell radius of $R$ and an area of $S$.

Based on the structure of HAPS communication system mentioned above, it is controlled by ideal power in a stationary state. The received power of each mobile station arriving at the HAPS base station is recorded as $P$, and the decimal expression of antenna gain is recorded as $G\left(\theta_{i j}\right)$, then the transmission power of the system can be expressed as

$$
P_{T}=P \cdot r_{i j}^{\mu} 10^{-\frac{G\left(\theta_{i j}\right)}{10}} \cdot 10^{-\frac{\varsigma_{i j}}{10}}
$$

Recording the voice activation factor as $\alpha$, it could be deduced that the system capacity of HAPS communication system is

$$
M=\frac{1}{\alpha(1+f)}\left[\frac{W / R_{b}}{E_{b} / I_{0}}-\frac{\sigma_{n}^{2}}{P}\right]+1
$$

where $I_{0}$ represents the total interference power and $\sigma_{n}^{2}$ is the variance of Gaussian noise, while $f$ denotes the interference factor which can be expressed as

$$
f=\frac{I_{\text {inter }}}{I_{\text {int ra }}}=\sum_{j \in T, j \neq 0} \int_{A} \frac{G\left(\theta_{0, i j}\right)}{G\left(\theta_{i j}\right)} \cdot \frac{1}{S} d A
$$

where $T$ is the area covered by a cluster, $I_{\text {intra }}, I_{\text {inter }}$ indicates interference within cell and inter-cells, respectively. In addition, their relationship could be illustrated as

$$
I_{\text {inter }}=\alpha(M-1) P
$$

In summary, the uplink capacity of HAPS communication system could be obtained. 


\subsection{Swing Effect}

Generally, wind in the stratosphere could be maintained in a certain direction for a long time. However, there is a big difference between platform swing and displacement. Among them, the displacement of the platform requires a certain time, and power equipment is required to ensure that it can stay at a certain position for a period of time, in an attempt to provide time guarantee for the switching. In fact, the platform swings at a certain angular velocity at all times and cannot stay at an angle [27]. Therefore, the paper assumes that the HAPS platform swings back and forth at an angle $\Delta \theta(t)$ away from the equilibrium point, as shown in Fig. 10.

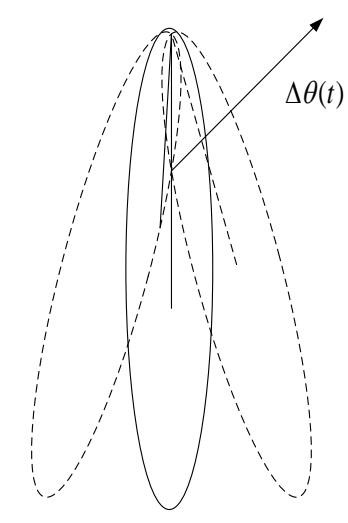

Fig. 10. Schematic diagram of platform swing

As shown in Fig. 10, the platform swings left and right within a certain range from the equilibrium point. The angle at which the platform deviates from the equilibrium point is recorded as $\Delta \theta(t)$, this is a Gaussian random variable with the mean of 0 and standard deviation of $\sigma$. In this way, the change of stratospheric platform could be expressed by the change of $\Delta \theta(t)$, and the average value of 0 indicates that the platform is in a quasi-static state. When the stratospheric platform is in a quasi-stationary state, the system capacity will be affected to a certain extent. Therefore, we will focus on analyzing the performance and capacity changes of HAPS when the platform swings at a small angle under the influence of wind. For the reason that there is currently no commercial stratospheric platform application system, it is not yet possible to give a precise platform specific motion mode. However, it is certain that the stratospheric platform could only be maintained in a quasi-stationary state where displacement and swing occur due to stratospheric wind with a speed of $-15 \mathrm{~m} / \mathrm{s}$ to $+15 \mathrm{~m} / \mathrm{s}$ under control of the platform [28]. The quasi-stationary state model under HAPS working state, swings in the range where the angle is less than 1 degree. Assume that the antenna angle of the array antenna remains the same when the platform is swinging, the swing model could be illustrated as Fig. 11. 


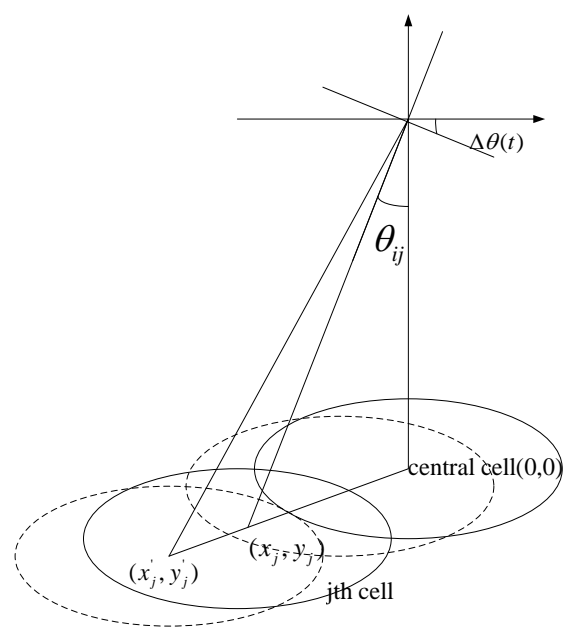

Fig. 11. HAPS swing model

It could be seen from Fig. 11, the center coordinate of the jth cell changes from original $\left(x_{j}, y_{j}\right)$ to $\left(x_{j}^{\prime}, y_{j}^{\prime}\right)$. Assume that the platform is swinging along the $\mathrm{x}$-axis direction, we can see that displacements of each cell in the $\mathrm{x}$-axis direction are the same which can be expressed as

$$
\left\{\begin{array}{c}
y_{j}^{\prime}=y_{j} \\
x_{j}^{\prime}=x_{j}+h \cdot \tan [\Delta \theta(t)]
\end{array}\right.
$$

The influence of the platform swing on the mobile station could be regarded as a kind of non-ideal power control, this paper considers that due to the randomness of the swing, the power control of the mobile station cannot keep up with the change of the swing, as a result the transmission power $P_{T}$ remains unchanged for a short time, which could be expressed as

$$
P_{T}=P \cdot r_{i j}^{\mu} 10^{-\frac{G\left(\theta_{i j}\right)}{10}} \cdot 10^{-\frac{S_{j}}{10}}
$$

When the platform is swinging, HAPS system capacity can be expressed as

$$
M=\frac{1}{\alpha\left(f_{\text {inter }}^{\prime}+f_{\text {intra }}^{\prime}\right)}\left[\frac{W / R_{b}}{E_{b} / I_{0}}-\frac{\sigma_{n}^{2}}{S}\right]+1
$$

where

$$
f_{\text {inter }}^{\prime}=\sum_{j \in T, j \neq 0} \int_{A} 10 \frac{G\left(\theta_{0, j}^{\prime}\right)-G\left(\theta_{i j}\right)}{10} \frac{1}{S} d A
$$

\section{Simulation Results and Analysis}

In this section, analysis will be made in regard to the effectiveness of proposed Markov chain-based channel model through simulations. Different coverage of stratospheric platform depending on elevation angle corresponds respectively to three areas which leads to three different channel states including Rice state, partially shaded state and fully shaded state. The 
simulation process could be illustrated as Fig. 12, where the input is randomly generated. To avoid ISI, the signal transmission rate is set to $200 \mathrm{~kb} / \mathrm{s}$, and then modulated by baseband DPSK. After that, the bit error rate (BER) could be count accordingly. The influence of these data signals on the transmission channel could be switched back and forth via the time ratio during a time block.

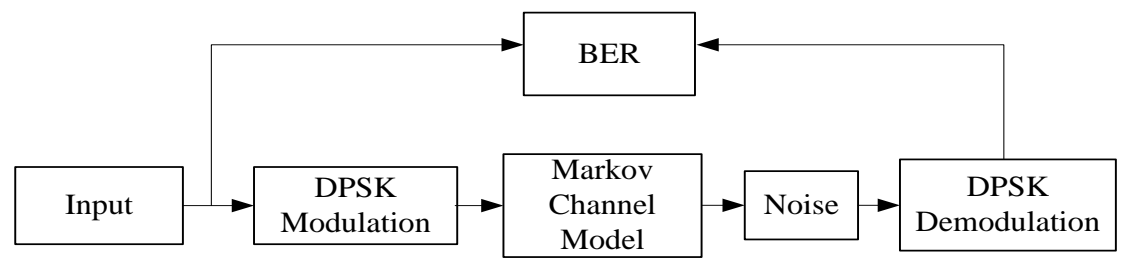

Fig. 12. Simulation block diagram

Just as shown in Fig. 12, we assume state $A$ is free from shadowing effect, that is, we could add direct signal with multipath signal, and its envelope complies with Rice distribution. Besides, for state $B$, its direct component of the received signal is affected by shadow occlusion, while the multipath component has no effect that follows Loo statistical probability model. Additionally, state $C$ is affected by shadow occlusion on its direct component and multipath component of the received signal, which could be demonstrated as Corazza statistical probability model in simulation scenario. Above all, we adopt Rice, Loo and Corazza as single static model. Therefore, to achieve these three states, certain parameters should be fitted. Specifically, state $A$ parameters determination could adopt Jakes approach, while the parameters of state $B$ could be fixed [29] in accordance with the actual data as

$$
\left\{\begin{array}{c}
\sigma_{0}^{2}(\partial)=B_{0}+B_{1} \partial+B_{2} \partial^{2}+B_{3} \partial^{3} \\
\mu(\partial)=\mu_{0}+\mu_{1} \partial+\mu_{2} \partial^{2}+\mu_{3} \partial^{3} \\
\sigma_{1}(\partial)=\sigma_{10}+\sigma_{11} \partial
\end{array}\right.
$$

For state $C$, that is fully shaded status, the parameters also could be fitted [30] through measured data in suburban environment as

$$
\left\{\begin{array}{c}
K(\partial)=K_{0}+K_{1} \partial+K_{2} \partial^{2} \\
\mu(\partial)=m_{0}+m_{1} \partial+m_{2} \partial^{1}+m_{3} \partial^{2} \\
\sigma_{1}(\partial)=\sigma_{01}+\sigma_{11} \partial
\end{array}\right.
$$

The performance of proposed channel model in different environments could be obtained through the simulation experiments on data blocks, and Fig. 13 and Fig. 14 correspond respectively to forests and suburbs environment, which illustrate the signal-to-noise (SNR), BER comparison curve of single-state and Markov chain-based channel model. 


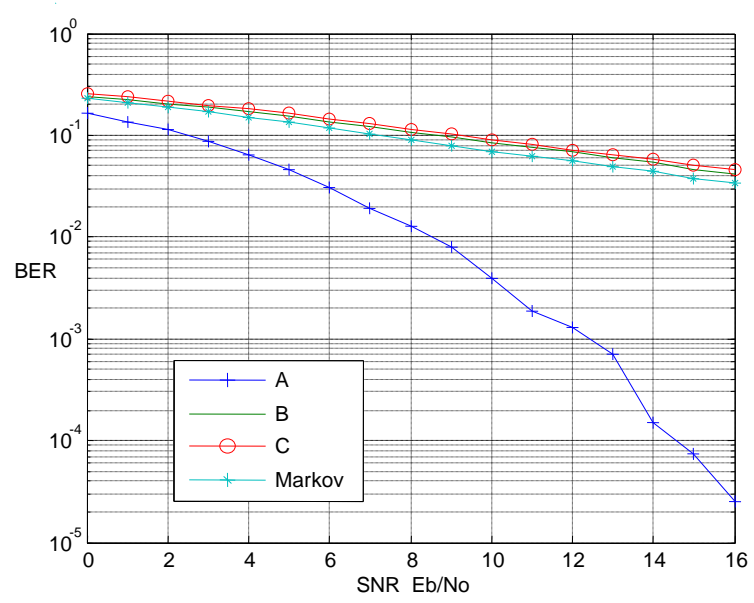

Fig. 13. Performance comparison under forest area

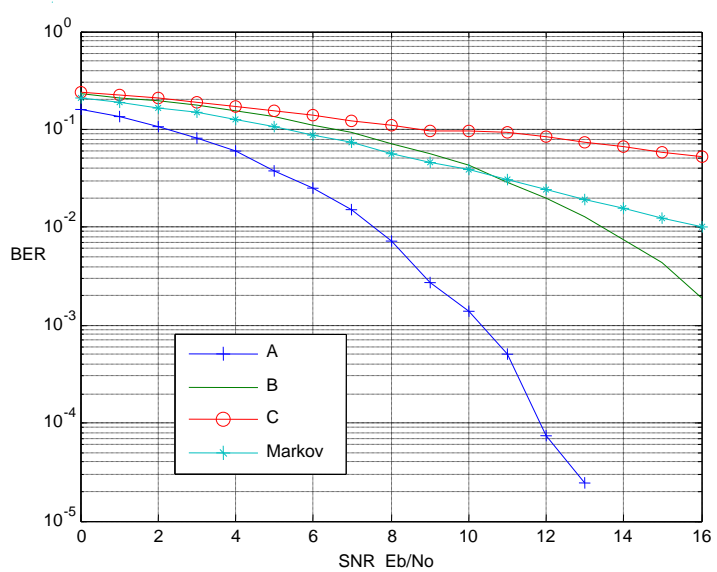

Fig. 14. Performance comparison in suburbs

In the Markov channel model, the BER of the system is between state $B$ and $C$, and this pattern is more obvious in forest areas. In the two figures above, the BER of state $A$ is lowest which is mainly influenced by shadowing effect, but it is just an ideal model because the increase in BER can also be overcome by channel coding. The SNR-BER comparison of suburban and forest areas are shown in Fig. 15. 


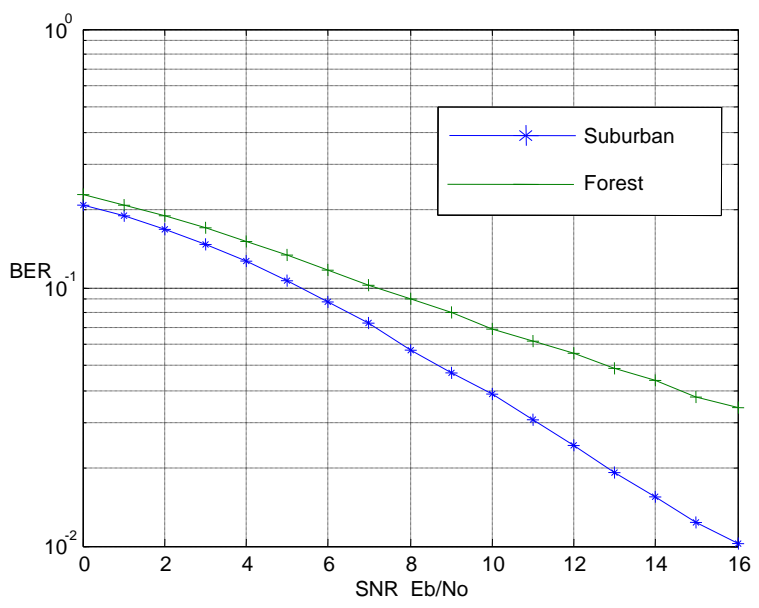

Fig. 15. Markov chain-based channel model performance comparison in different regions

In Fig. 15, it can be observed that the BER of HAPS communication system in forest area is higher than that in suburbs. Therefore, in suburbs the shadow shielding effect is much smaller and the channel transmission performance is better than that in forest area. In addition, the cellular structure model of HAPS system is used when analyzing the changes in cell interference factors and system capacity under platform swing. Among them, the reference cell for main beam alignment is located at the center of layered structure, and its main parameters are as shown in Table 3.

Table 3. Parameter values

\begin{tabular}{|c|c|}
\hline Parameter & Value \\
\hline$R$ & $1 \mathrm{~km}$ \\
\hline$\alpha$ & 0.6 \\
\hline$W / R_{b}$ & 256 \\
\hline$E_{b} / I_{0}$ & $5 d B$ \\
\hline$P / \sigma_{n}^{2}$ & $-1 d B$ \\
\hline
\end{tabular}

When the HAPS platform swings to a maximum angle of $1^{\circ}$, the influence of different cell circle numbers on the system capacity is shown in Fig. 16. 


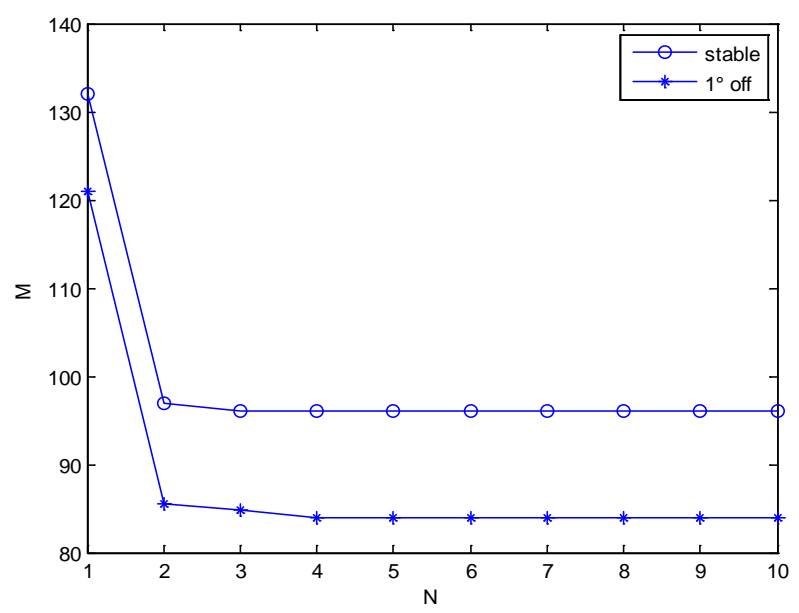

Fig. 16. Uplink capacity comparison

It could be seen from Fig. 16, when swinging to $1^{\circ}$, the system capacity is greatly reduced. Among them, when the number of cell circles is $N=1$, the capacity of HAPS system in stationary state is 132 , while the swing state is reduced to 121 , which decreases the capacity by $8.3 \%$. In addition, when the number of cell circles is $N=10$, the system capacity in the static state is 96 . At the same time, the capacity of platform swing to $1^{\circ}$ is reduced to 84 , which is a $12.5 \%$ decrease. Driven from the results analysis above, the impact of swing on HAPS system's capacity should not be ignored in reality. Therefore, we need to take swing effect into account under such kind of scenario assumptions, instead of simply assuming that it has no effect.

\section{Conclusion}

To support the rapid growth of large-scale IoT services and applications, this paper proposes an effective channel model in HAPS communication based on Markov chain. Afterwards, the uplink capacity of HAPS communication system is analyzed. Besides, to cope with swing effect, the proposed scheme establishes a swing model that obeys zero mean Gaussian distribution. Finally, the effectiveness of proposed method is verified with simulation results.

\section{Acknowledgement}

This work was supported in part by the China Scholarship Council under Grant 20173109, in part by the National Natural Science Foundation of China under Grant 61901049, and in part by the Fundamental Research Funds for Central Universities.

\section{References}

[1] C. Zhu, J. J. P. C. Rodrigues, V. C. M. Leung, L. Shu, and L. T. Yang, "Trust-Based Communication for the Industrial Internet of Things,” IEEE Communications Magazine, vol. 56, no. 2, pp. 16-22, February 2018. Article (CrossRef Link) 
[2] Emil Björnson, E. G. Larsson and T. L. Marzetta, "Massive MIMO: ten myths and one critical question," IEEE Communications Magazine, vol. 54, no. 2, pp. 114-123, February 2016. Article (CrossRef Link)

[3] C. Zhu, V. C. M. Leung, K. Wang, L. T. Yang, and Y. Zhang, "Multi-Method Data Delivery for Green Sensor-Cloud," IEEE Communications Magazine, vol. 55, no. 5, pp. 176-182, May 2017. Article (CrossRef Link)

[4] G. Karabulut Kurt et al., "A Vision and Framework for the High Altitude Platform Station (HAPS) Networks of the Future," IEEE Communications Surveys \& Tutorials, vol. 23, no. 2, pp. 729-779, Secondquarter 2021. Article (CrossRef Link)

[5] S. Zhang, W. Quan, J. Li, W. Shi, P. Yang and X. Shen, "Air-Ground Integrated Vehicular Network Slicing with Content Pushing and Caching," IEEE Journal on Selected Areas in Communications, vol. 36, no. 9, pp. 2114-2127, September 2018. Article (CrossRef Link)

[6] Y. Shibata, N. Kanazawa, M. Konishi, K. Hoshino, Y. Ohta and A. Nagate, "System Design of Gigabit HAPS Mobile Communications," IEEE Access, vol. 8, pp. 157995-158007, 2020. Article (CrossRef Link)

[7] M. Guan et al., "Efficiency Evaluations Based on Artificial Intelligence for 5G Massive MIMO Communication Systems on High-Altitude Platform Stations," IEEE Transactions on Industrial Informatics, vol. 16, no. 10, pp. 6632-6640, October 2020. Article (CrossRef Link)

[8] X. Cao, P. Yang, M. Alzenad, X. Xi, D. Wu and H. Yanikomeroglu, "Airborne Communication Networks: A Survey," IEEE Journal on Selected Areas in Communications, vol. 36, no. 9, pp. 1907-1926, September 2018. Article (CrossRef Link)

[9] K. Hoshino, S. Sudo and Y. Ohta, "A Study on Antenna Beamforming Method Considering Movement of Solar Plane in HAPS System," in Proc. of the 90th IEEE Vehicular Technology Conference, pp. 1-5, 2019. Article (CrossRef Link)

[10] M. D. Zakaria, D. Grace, P. D. Mitchell, T. M. Shami and N. Morozs, "Exploiting User-Centric Joint Transmission - Coordinated Multipoint With a High Altitude Platform System Architecture," IEEE Access, vol. 7, pp. 38957-38972, 2019. Article (CrossRef Link)

[11] A. M. Al-Awadi and M. J. Al-Dujaili, "Simulation of LTE-TDD in the HAPS Channel," International Journal of Electrical and Computer Engineering, vol. 10, no. 3, pp. 3152-3157, 2020. Article (CrossRef Link)

[12] S. Zhang, W. Quan, J. Li, W. Shi, P. Yang and X. Shen, "Air-Ground Integrated Vehicular Network Slicing With Content Pushing and Caching," IEEE Journal on Selected Areas in Communications, vol. 36, no. 9, pp. 2114-2127, 2018. Article (CrossRef Link)

[13] G. Kurt, M. G. Khoshkholgh, S. Alfattani, A. Ibrahim, T. S. Darwish, M. S. Alam, H. Yanikomeroglu and A. Yongacoglu, "A vision and framework for the high altitude platform station (HAPS) networks of the future," Preprint. arXiv:2007.15088, 2007.

[14] K. Tekbıyık, G. K. Kurt, C. Huang, A. R. Ekti and H. Yanikomeroglu, "Channel Estimation for Full-Duplex RIS-assisted HAPS Backhauling with Graph Attention Networks," in Proc. of ICC 2021-IEEE International Conference on Communications, pp. 1-6, 2021. Article (CrossRef Link)

[15] Technical and Operational Characteristics for the Fixed Service Using High Altitude Platform Stations in the Bands 27.5- 28.35 GHz and 31-31.3 GHz, ITU-RF.1569.

[16] O. Anicho, P. B. Charlesworth, G. S. Baicher, A. Nagar and N. Buckley, "Comparative Study for Coordinating Multiple Unmanned HAPS for Communications Area Coverage," in Proc. of the 2019 International Conference on Unmanned Aircraft Systems, pp. 467-474, 2019. Article (CrossRef Link)

[17] C. Queiroz, R. Vieira, A. Barreto, A. Zarrebini, E. Souza and A. Linhares, "New Spectrum Bands for HAPS: Sharing with Fixed-Satellite Systems," in Proc. of the 89th IEEE Vehicular Technology Conference, pp. 1-5, 2019. Article (CrossRef Link)

[18] O. Anicho, P. B. Charlesworth, G. S. Baicher and A. Nagar, "Autonomously Coordinated Multi-HAPS Communications Network: Failure Mitigation in Volcanic Incidence Area Coverage," in Proc. of the 2019 IEEE International Conference on Communication, Networks and Satellite, pp. 79-84, 2019. Article (CrossRef Link) 
[19] M. Bacco, et al., "IoT Applications and Services in Space Information Networks," IEEE Wireless Communications, vol. 26, no. 2, pp. 31-37, April 2019. Article (CrossRef Link)

[20] S. Dutta, F. Hsieh and F. W. Vook, "HAPS Based Communication using mmWave Bands," in Proc. of the 2019 IEEE International Conference on Communications, pp. 1-6, 2019. Article (CrossRef Link)

[21] M.S. Alam, G. K. Kurt, H. Yanikomeroglu, P. Zhu, and N. D. Đào, "High Altitude Platform Station based Super Macro Base Station Constellations," Preprint. arXiv: 2007.08747, 2007.

[22] T. S. Rapp port, Wireless Communication Principles and Practice, London: Prentice Hall PTR, 2006.

[23] W. Guo, W. Zhang, P. Mu, F. Gao and H. Lin, "High-Mobility Wideband Massive MIMO Communications: Doppler Compensation, Analysis and Scaling Laws," IEEE Transactions on Wireless Communications, vol. 18, no. 6, pp. 3177-3191, June 2019. Article (CrossRef Link)

[24] R. Swaminathan, S. Sharma and A. S. MadhuKumar, "Performance Analysis of HAPS-Based Relaying for Hybrid FSO/RF Downlink Satellite Communication," in Proc. of the 91st IEEE Vehicular Technology Conference, pp. 1-5, 2020. Article (CrossRef Link)

[25] T. Fujii, Y. Ohta and T. Fujii, "A Study on Signal Band Division Interference Canceller for HAPS Feeder Links with Multi-Gateways," in Proc. of the 91st IEEE Vehicular Technology Conference, pp. 1-7, 2020. Article (CrossRef Link)

[26] K. Hoshino, S. Sudo and Y. Ohta, "A Study on Antenna Beamforming Method Considering Movement of Solar Plane in HAPS System," in Proc. of the 90th IEEE Vehicular Technology Conference, pp. 1-5, 2019. Article (CrossRef Link)

[27] C. Queiroz, R. Vieira, A. Barreto, A. Zarrebini, E. Souza and A. Linhares, "New Spectrum Bands for HAPS: Sharing with Fixed-Satellite Systems," in Proc. of the 89th IEEE Vehicular Technology Conference, 2019. Article (CrossRef Link)

[28] M. Konishi, T. Nishimaki, Y. Shibata, S. Nabatame and A. Nagate, "A Study of Co-Channel Spectrum-Sharing System between HAPS and Terrestrial Mobile Communication Networks," in Proc. of the 91st IEEE Vehicular Technology Conference, pp. 1-5, 2020. Article (CrossRef Link)

[29] A. Neul, J. Hagenauer, W. Papke, F. Dolainsky and F. Edbauer, "Aeronautical Channel Characterization Based on Measurement Flights," in Proc. of the Global Telecommunications Conference, 1987.

[30] A. Neul, Modulation and Codierung im aeronautischen Satellitenkanal, University of the Federal Armed Forces Munich, 1989.

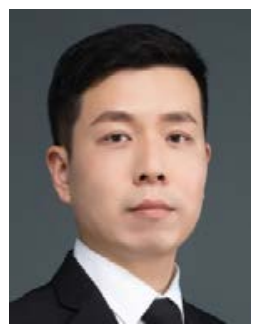

Wei Yang received the Ph.D. degree in Information and Communication Engineering from Beijing University of Posts and Telecommunications (BUPT), Beijing, China, in 2020. He is currently a Research Fellow with the State Key Laboratory of Networking and Switching Technology, BUPT, China. He is also a Postdoctoral Researcher with the Guangdong Southern Planning \& Designing Institute of Telecom Consultation Co., Ltd., Shenzhen, China. From 2018 to 2019, he was a Visiting Scholar at the Department of Computer Science and Engineering, Santa Clara University, CA, USA. His research interests include wireless communication, cyber-physical system and information security.

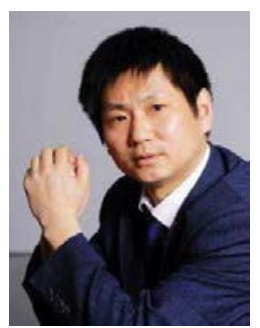

Xiaojun Jing received the B.S. degree from Beijing Normal University, and the M.S. and Ph.D. degrees from National University of Defense Technology in 1995 and 1999, respectively. From 2000 to 2002, he was a Postdoctoral Researcher with Beijing University of Posts and Telecommunications (BUPT), Beijing, China, where he is currently a Full Professor with the School of Information and Communication Engineering. His research interests include wireless communication, information security and image processing. 


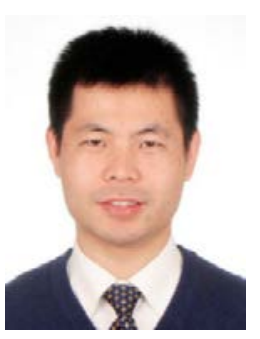

Hai Huang received the Ph.D. degree from Beihang University and was a Postdoctoral Researcher at State Key Laboratory of Virtual Reality Technology and Systems. He is currently an Associate Professor of Information and Communication Engineering at Beijing University of Posts and Telecommunications. His research interests include wireless communication, virtual reality and pattern recognition.

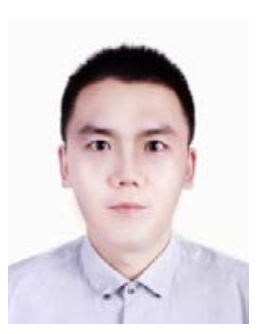

Chunsheng Zhu is an Associate Professor in the Institute of Future Networks at Southern University of Science and Technology in China. He is also an Associate Researcher in the Research Center of Networks and Communications at Peng Cheng Laboratory in China. He received the Ph.D. Degree in Electrical and Computer Engineering from The University of British Columbia, Canada. He has authored more than 100 publications published or accepted by refereed international journals (e.g., IEEE Transactions on Industrial Electronics, IEEE Transactions on Computers, IEEE Transactions on Information Forensics and Security, IEEE Transactions on Mobile Computing, IEEE Transactions on Industrial Informatics, IEEE Transactions on Wireless Communications, IEEE Transactions on Vehicular Technology, IEEE Transactions on Emerging Topics in Computing, IEEE Transactions on Cloud Computing, ACM Transactions on Embedded Computing Systems, ACM Transactions on Cyber-Physical Systems), magazines (e.g., IEEE Communications Magazine, IEEE Wireless Communications Magazine, IEEE Network Magazine), and conferences (e.g., IEEE INFOCOM, IEEE IECON, IEEE SECON, IEEE DCOSS, IEEE ICC, IEEE GLOBECOM). His research interests mainly include Internet of Things, wireless sensor networks, cloud computing, big data, social networks, and security.

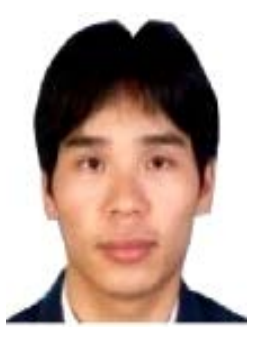

Qiaojie Jiang is a Senior Engineer at the Guangdong Southern Planning \& Designing Institute of Telecom Consultation Co., Ltd., Shenzhen, China. He received the M.S. degree from Nanjing University of Posts and Telecommunications, Nanjing, China, and has mainly been engaged in communication engineering management and research on new mobile communication technologies in recent years. His research interests include wireless network planning technology, Internet of Things deployment and application.

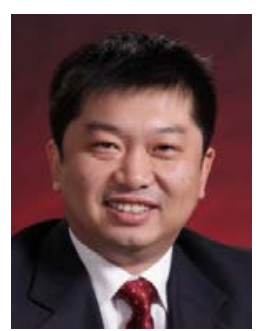

Dongliang Xie received the Ph.D. degree from Beijing Institute of Technology, Beijing, China, in 2002. He was a Visiting Researcher in the Department of Electrical and Computer Engineering, State University of New York at Stony Brook. He is currently a Full Professor with the State Key Laboratory of Networking and Switching Technology, Beijing University of Posts and Telecommunications, Beijing, China. His research interests focus on resource-constrained wireless communication and information-centric network, including architecture of ubiquitous and heterogeneous network, complex network analysis, as well as content retrieval and service management. 